\title{
TeV Cosmic Ray Anisotropy and the Heliospheric Magnetic Field
}

\author{
P. Desiati ${ }^{1,2}$ and A. Lazarian ${ }^{2}$ \\ ${ }^{1}$ Wisconsin IceCube Particle Astrophysics Center (WIPAC), University of Wisconsin, Madison, \\ WI 53703, USA \\ ${ }^{2}$ Department of Astronomy, University of Wisconsin, Madison, WI 53706, USA \\ Correspondence to: P. Desiati (desiati@wipac.wisc.edu)
}

Received: 7 May 2014 - Revised: 13 August 2014 - Accepted: 14 August 2014 - Published: 31 October 2014

\begin{abstract}
Cosmic rays are observed to possess a small non uniform distribution in arrival direction. Such anisotropy appears to have a roughly consistent topology between tens of $\mathrm{GeV}$ and hundreds of $\mathrm{TeV}$, with a smooth energy dependency on phase and amplitude. Above a few hundreds of $\mathrm{TeV}$ a sudden change in the topology of the anisotropy is observed. The distribution of cosmic ray sources in the Milky Way is expected to inject anisotropy on the cosmic ray flux. The nearest and most recent sources, in particular, are expected to contribute more significantly than others. Moreover the interstellar medium is expected to have different characteristics throughout the Galaxy, with different turbulent properties and injection scales. Propagation effects in the interstellar magnetic field can shape the cosmic ray particle distribution as well. In particular, in the 1-10 TeV energy range, they have a gyroradius comparable to the size of the Heliosphere, assuming a typical interstellar magnetic field strength of $3 \mu \mathrm{G}$. Therefore they are expected to be strongly affected by the Heliosphere in a manner ordered by the direction of the local interstellar magnetic field and of the heliotail. In this paper we discuss on the possibility that $\mathrm{TeV}$ cosmic rays arrival distribution might be significantly redistributed as they propagate through the Heliosphere.
\end{abstract}

In the last few decades a number of experiments have provided long term and high statistical significance evidence of a global anisotropy in the cosmic ray distribution with a relative amplitude of order $10^{-4}-10^{-3}$. Observations in the Northern Hemisphere were reported from energies of tens to several hundreds of $\mathrm{GeV}$ with muon detectors (Nagashima et al., 1998; Hall et al., 1999; Munakata et al., 2010), in the one to tens of $\mathrm{TeV}$ energy range with various surface arrays and underground detectors (Amenomori et al., 2005, 2006, 2011; Guillian et al., 2007; Abdo et al., 2009; Amenomori et al., 2009; Cui, 2011; Di Sciascio, 2013; de Jong, 2011; BenZvi et al., 2013; Santander et al., 2013a), up to hundreds of TeV (Aglietta et al., 2009). Recently, similar observations of a large angular scale anisotropy were reported in the Southern Hemisphere by the IceCube Observatory, in the energy range between $10 \mathrm{TeV}$ and a few $\mathrm{PeV}$ (Abbasi et al., 2010, 2012; Aartsen et al., 2013). These results show that the anisotropy matches the observations in the Northern Hemisphere and that a sudden change in its topology occurs at energy in excess of a few hundreds of $\mathrm{TeV}$, confirming the observations by Aglietta et al. (2009). The transition between the two anisotropy regimes is preceded by a steady decrease of the amplitude at particle energy above $10 \mathrm{TeV}$, following an increase trend at lower energies (see Amenomori et al., 2005 for instance). The global anisotropy cannot be described with a simple dipole, but as a superposition of spherical harmonic contributions, where statistically significant small angular scale features, with amplitude of order $10^{-5}-10^{-4}$, are also observed (Abbasi et al., 2011; Santander et al., 2013a), in agreement with similar observations in the Northern Hemisphere (Amenomori et al., 2007; Abdo et al., 2008; Bartoli et al., 2013; BenZvi et al., 2013), as shown in Fig. 1. See BenZvi (2014) for more information. 
The origin of the observed anisotropy is not understood yet. However it is reasonable to assume that it is a combination of effects correlated to the distribution of the galactic sources of cosmic rays, of the geometry and turbulent properties of the galactic magnetic field and on propagation. These are likely also responsible for the complex shape of the energy spectrum (Gaisser et al., 2013). Since we don't know where the sources of cosmic rays are and we don't know the details of the interstellar magnetic field, explaining the observations is not an easy task.

The large scale anisotropy can be qualitatively explained on the basis of diffusive propagation of cosmic rays in the Milky Way from stochastically distributed sources. Numerical studies show that it is possible to find a given realization of galactic source distribution that might explain the observed non monotonic energy dependence of the anisotropy amplitude, although for an ensemble of realizations the mean amplitude is overestimated compared to observations (Erlykin and Wolfendale, 2006; Blasi and Amato, 2012; Ptuskin, 2012; Pohl and Eichler, 2013; Sveshnikova et al., 2013). Even accounting for a more likely anisotropic diffusion, observations appear to be outliers compared to predictions (Effenberger et al., 2012; Kumar and Eichler, 2014). The fact that cosmic ray anisotropy is not a simple dipole but can be mostly explained with a superposition of a dipole and quadrupole term, seems to suggest that other transport processes might be important as well. For instance drift diffusion driven by a gradient of cosmic ray density in the local interstellar medium, producing a bi-directional anisotropy, was considered by Amenomori et al. (2007) and Mizoguchi et al. (2009).

The small scale anisotropy may be produced by the interactions of cosmic rays with an isotropically turbulent interstellar magnetic field. Scattering processes with stochastic magnetic instabilities produce perturbations in the arrival direction distribution of an anisotropic distribution of cosmic ray particles within the scattering mean free path. Such perturbations may be observed as stochastic localized excess or deficit regions (Giacinti and Sigl, 2012; Biermann et al., 2012), and the corresponding angular power spectrum can be analytically predicted from the Liouville Theorem (Ahlers, 2014). The injection scale of interstellar turbulence is of order $10 \mathrm{pc}$ within the galactic arms and $100 \mathrm{pc}$ in the inter arm regions (Haverkorn et al., 2006). In the cascading processes down to smaller scales, the turbulent eddies become elongated along the magnetic field lines. This anisotropic turbulence makes scattering processes inefficient, and scattering mean free path can be larger than turbulence injection scale, so that particles basically stream along magnetic field lines with small cross fieldline transport (Yan and Lazarian, 2008; Lazarian and Yan, 2013).

Besides the cascading interstellar magnetic field turbulence down to damping scale (typically of order $0.1 \mathrm{pc}$ ), there are other sources of magnetic perturbations on smaller scales. The closest to Earth is represented by the Heliosphere, formed by the interaction between the solar wind and the interstellar flow. It is about 600 astronomical units (AU) wide and it can extend several thousands AU downstream the interstellar wind (Pogorelov et al., 2009). Globally, the Heliosphere constitutes a perturbation in the $3 \mu \mathrm{G}$ Local Interstellar Magnetic Field (LIMF) with injection scale comparable $\sim 10 \mathrm{TeV}$ proton gyroradius. It is therefore reasonable that the LIMF draping around the Heliosphere might be a significant source of resonant scattering, capable of redistributing the arrival directions of $\mathrm{TeV}$ cosmic cosmic ray particles.

In this paper we briefly summarize the current understanding of the effects of the Heliosphere on $\mathrm{TeV}$ cosmic rays and whether we can use the anisotropy observations as a probe into the properties of the heliospheric boundary with the local interstellar medium.

\section{The Heliosphere}

The solar system is located approximately $8 \mathrm{kpc}$ from the galactic center and it takes part in the global galactic rotation, similarly to the Local Interstellar Medium (LISM). However, there is a relative motion of the solar system with respect to the LISM. This is determined by the expansion of the Loop I superbubble from the Scorpion-Centaurus Association (at a distance of about $60 \mathrm{pc}$ ), which is near the apparent direction of the galactic center (Frisch et al, 2011). The solar system is currently located within an expanding shell of Loop I, which is breaking up in smaller cloudlets that share similar composition and velocity. And the LIMF appears to be almost parallel to the shell front and relatively coherent within a distance of order $100 \mathrm{pc}$. One of these cloudlets, the so called Local Interstellar Cloud (LIC) is where the solar system is located, and it moves at a velocity of approximately $23 \mathrm{~km} \mathrm{~s}^{-1}$ with respect to the Sun (McComas et al., 2012). The interaction of the supersonic outward solar wind and the incoming interstellar flow produced a comet shaped interface where the interplanetary and the interstellar magnetic fields rearrange. At a distance of about 80 AU from Earth the termination shock is formed where the solar wind becomes subsonic, but the separation between the heliospheric and the interstellar medium occurs at a distance of about $120 \mathrm{AU}$ in the upstream direction, where the heliopause is located. From numerical simulations, the Heliosphere is expected to be as thick as $600 \mathrm{AU}$ and to form an elongated tail in the downstream direction that can be as long as several thousand $\mathrm{AU}$ (Pogorelov et al., 2009).

Since the LISM is a partially ionized medium, neutral particles can penetrate through the heliopause and have charge exchange with the highly ionized solar wind. Such processes create instabilities on the heliopause in the upstream direction, that propagate along the flanks and that can reach spatial scales of 10-100 AU (Borovikov, 2008). Another source of instabilities on the heliopause is the solar cycles affecting the heliospheric magnetic field pressure that determines the 
location of the heliopause (Pogorelov et al., 2013). The magnetic field polarity inversions due to solar cycles, also produce unipolar regions of magnetic fields that, dragged by the solar wind, drift along the heliotail (Pogorelov et al., 2009).

\section{Heliospheric influence on $\mathrm{TeV}$ cosmic rays}

Globally, the Heliosphere is a perturbation of the LIMF on a scale of about $600 \mathrm{AU}$. In addition, complex structures in the inner heliospheric magnetic field of about 100-200 AU and instabilities on the heliopause of approximately 10-100 AU, have necessarily an effect on the charged particles that propagate through.

In Desiati and Lazarian (2013) the scenario of $\mathrm{TeV}$ cosmic ray interaction with the Heliosphere was first presented as a possible cause of the observed anisotropy. It is known that galactic cosmic rays below $100 \mathrm{GeV}$ are strongly influenced by solar activity and by the geometry and small scale instabilities (sub-AU scale) of the interplanetary magnetic field within the termination shock. In the TeV energy scale such small scale effects are subdominant because the gyroradius of a proton is larger than the termination shock size, in the inner heliospheric magnetic field. However TeV cosmic rays are in gyro-resonance with the large scale heliospheric magnetic field, with unipolar magnetic regions in the inner heliotail and with the instabilities on the boundary with the LISM, depending on the local pitch angle. As a consequence, an incoming flux of $\mathrm{TeV}$ cosmic rays with an initial anisotropy (e.g. induced by uneven distribution of galactic sources) may be subject to a significant redistribution of arrival direction when crossing the Heliosphere, even without affecting the amplitude of the anisotropy.

Figure 1 shows the sky map of the relative intensity of the global cosmic ray anisotropy (on the top) and the sky map of statistical significance of the small scale anisotropy (on the bottom) obtained by filtering out all the cosmic ray count gradients compatible with a dipole and a quadrupole. In both maps the observations from IceCube, at median particle energy of $20 \mathrm{TeV}$, are shown. In the Northern Hemisphere the figures show the results from Tibet-AS $\gamma$ (with median energy of $5 \mathrm{TeV}$ ) and from Milagro (with median energy of about $1 \mathrm{TeV}$ ). The figures show a degree of matching compatibility between the different observations from the Northern and Southern Hemispheres, the differences possibly attributed to the different sensitivities of the cited experiments on the energy and, possibly, mass composition of the cosmic rays. Some visible features seem ordered by the LIMF (with three direction estimates from Funsten et al., 2009; Schwadron et al., 2009; Heerikhuisen et al., 2010), in particular the fact that the magnetic equator "at infinity" (i.e. the plane perpendicular to the unperturbed LIMF and passing by Earth, and represented with black lines in Fig. 1) seems to be aligned with the boundary region between the excess and the deficit of the anisotropy. But there appears to be some

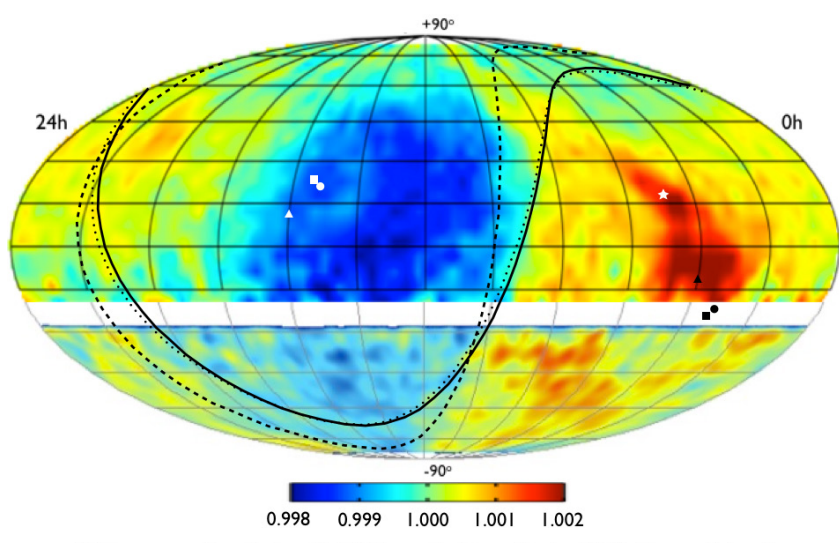

Milagro + IceCube TeV Cosmic Ray Data ( $10^{\circ}$ Smoothing)

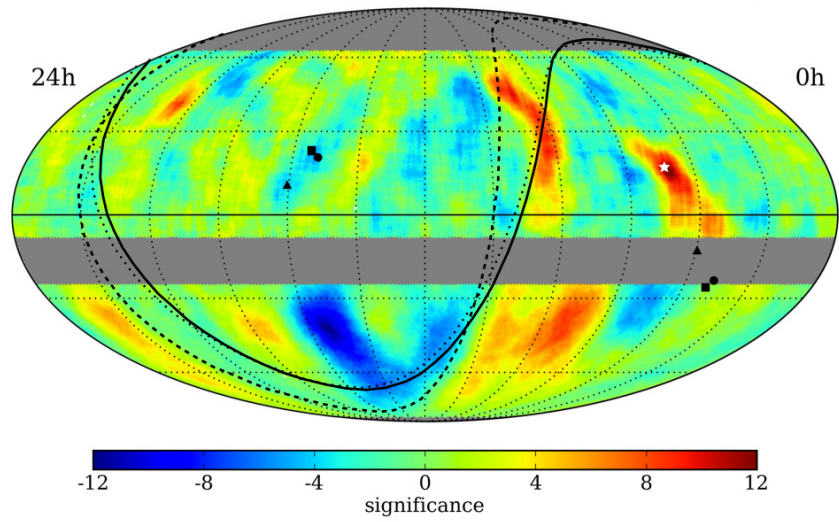

Figure 1. Top: map in equatorial coordinates constructed by combining relative intensity distributions of cosmic ray counts independently normalized within declination bands of order $1^{\circ}-5^{\circ}$. The map shows the observation by Tibet AS $\gamma$ at about $5 \mathrm{TeV}$ in the Northern Hemisphere (Amenomori et al., 2011) and by IceCube at a median energy of $20 \mathrm{TeV}$ in the Southern Hemisphere Abbasi et al. (2010). Bottom: map in equatorial coordinates of the statistical significance of the observed cosmic ray counts. The map shows the observation by Milagro at about 1fig01.pdfTeV in the Northern Hemisphere (from Abdo et al., 2008) and that by the IceCube Observatory at a median energy of $20 \mathrm{TeV}$ in the Southern Hemisphere (from Abbasi et al., 2011). Also shown the equator "at infinity" (see text) in black continuous line, corresponding to the direction of LIMF inferred in Funsten et al. (2009, circular symbols), that in black dotted line from Schwadron et al. (2009, square symbols), and that in black dashed line from Heerikhuisen et al. (2010, triangle symbols). The star symbol indicates the downwind interstellar medium flow with coordinate $\left(5 \mathrm{~h},+17^{\circ}\right)$. Color scale indicates relative excess (red) and deficit (blue) with respect to average intensity. From Desiati and Lazarian (2013).

ordering by the heliotail direction as well (almost coinciding with the downstream interstellar medium flow direction, represented with a white star in Fig. 1).

At $10 \mathrm{TeV}$, cosmic ray protons streaming along the LIMF have gyroradii comparable to the heliospheric size, thus making it possible for them to undergo resonant scattering. Thus $10 \mathrm{TeV}$ energy scale represents a transition scale between 


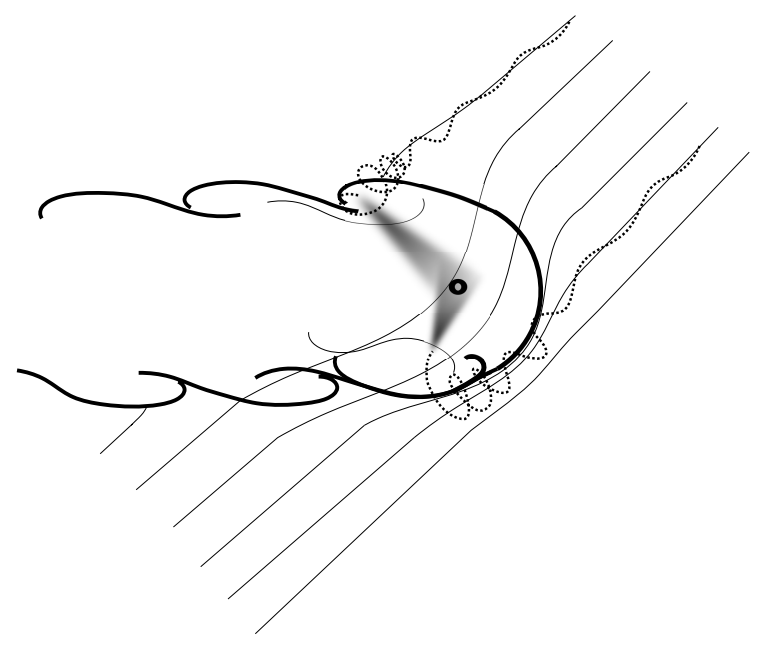

Figure 2. Illustration of the turbulent ripples at large scale on the outer heliospheric boundary. High energy cosmic rays propagating from the upstream direction into the front of the Heliosphere resonantly scatter with the trailing magnetic field perturbations on the flanks of the Heliosphere and along the heliotail. The uniform pitch angle distribution makes it possible for particles to scatter back to Earth. The grey blurred area schematically represents the possible scattered particle trajectories toward Earth.

the lower energies, where cosmic rays follows adiabatically the magnetic field lines, and the higher energies, where the heliospheric influence starts to become subdominant. This is in general agreement with the energy dependence of the anisotropy amplitude that increases up to about $10 \mathrm{TeV}$ and decreases above this energy (Amenomori et al., 2005). Resonant scattering may be able to redistribute the $10^{-3}$ interstellar anisotropic component of the cosmic ray arrival distribution, depending on particle energy and pitch angle, and on the properties of heliospheric magnetic field inside the tail and on the heliopause. Backscattering on the magnetic instabilities at the boundary with the LISM (see Fig. 2 for a simple illustration) may have the effect to locally amplify particle density gradients which can be interpreted as localized features (Desiati and Lazarian, 2013).

The localized excess region of cosmic rays observed in the direction of the heliotail, highly pronounced in the sub-TeV energy range (Nagashima et al., 1998), seems to be characterized by a harder than average energy spectrum up to about $10 \mathrm{TeV}$ (Abdo et al., 2008; Bartoli et al., 2013). If confirmed, such observations might indicate that the small fraction of cosmic rays from this narrow angular region might be reaccelerated in magnetic reconnection processes occurring in between the unipolar magnetic domains generated by solar cycles (Lazarian and Desiati, 2010; Desiati and Lazarian, 2012). Such reacceleration processes would only involve a negligible fraction of the total turbulent energy transported by the solar wind across the heliotail.
In Schwadron et al. (2014) numerical calculations show that $\mathrm{TeV}$ cosmic ray arrival direction distribution, originally ordered by the LIMF, is perturbed so that they appear to be ordered by their entry into the Heliosphere, assuming the direction of the interstellar medium flow velocity and of the LIMF as evaluated from the analysis of data by the Interstellar Boundary Explorer (IBEX). A similar result was obtained by Zhang et al. (2014). If a dipole anisotropy is assumed, either from the contribution of a nearby recent source of cosmic rays or simply from the convective gradient due to the relative motion with respect to the LISM, the deformation induced by the LIMF draping around the Heliosphere was estimated and found to globally resemble the actual observations.

Another heliospheric scenario appeals to the possibility that electric fields arising from the motion of the heliospheric plasma might be able to produce the observed localized excess regions of $\mathrm{TeV}$ cosmic rays (Drury, 2013).

In conclusion, the effect of the Heliosphere in the local interstellar magnetic field, produces resonant scattering processes on $10 \mathrm{TeV}$ scale cosmic protons. Depending on the instabilities of the heliospheric magnetic fields, such resonant processes may be strong enough to redistribute the arrival direction of cosmic rays from an initial anisotropic distribution. This is quite a different scenario that the very recent studies where it was found that the Heliosphere has only a perturbative effects on $\mathrm{TeV}$ cosmic rays. The authors are studying this phenomenon in great details, utilizing the state-of-the-art heliospheric modeling including magnetic instabilities. Results will be published soon.

Acknowledgements. P. Desiati acknowledges the support from the U.S. National Science Foundation-Office of Polar Programs. A. Lazarian acknowledges the support of the NSF grant AST1212096, NASA grant X5166204101, and of the NSF-sponsored Center for Magnetic Self-Organization.

Edited by: J. Tjus

Reviewed by: R. Tautz and one anonymous referee

\section{References}

Aartsen, M. G., Abbasi, R., Abdou, Y., et al. (IceCube Collaboration): Observation of Cosmic-Ray Anisotropy with the IceTop Air Shower Array, Astrophys. J., 765, 55, doi:10.1088/0004637X/765/1/55, 2013.

Abbasi, R., Abdou, Y., Abu-Zayyad, T., et al.: Measurement of the Anisotropy of Cosmic-ray Arrival Directions with IceCube, Astrophys. J. Lett., 718, L194, doi:10.1088/2041-8205/718/2/L194, 2010.

Abbasi, R., Abdou, Y., Abu-Zayyad, T., et al.: Observation of Anisotropy in the Arrival Directions of Galactic Cosmic Rays at Multiple Angular Scales with IceCube, Astrophys. J., 740, 16, doi:10.1088/0004-637X/740/1/16, 2011. 
Abbasi, R., Abdou, Y., Abu-Zayyad, T., et al.: Observation of Anisotropy in the Galactic Cosmic-Ray Arrival Directions at 400 $\mathrm{TeV}$ with IceCube, Astrophys. J., 746, 33, doi:10.1088/0004637X/746/1/33, 2012.

Abdo, A. A., Allen, B., Aune, T., Berley, D., Blaufuss, E., Casanova, S., Chen, C., Dingus, B. L., Ellsworth, R. W., Fleysher, L., Fleysher, R., Gonzalez, M. M., Goodman, J. A., Hoffman, C. M., Hüntemeyer, P. H., Kolterman, B. E., Lansdell, C. P., Linnemann, J. T., McEnery, J. E., Mincer, A. I., Nemethy, P., Noyes, D., Pretz, J., Ryan, J. M., Parkinson, P. M. Saz, Shoup, A., Sinnis, G., Smith, A. J., Sullivan, G. W., Vasileiou, V., Walker, G. P., Williams, D. A., and Yodh, G. B. (Milagro Collaboration): Discovery of Localized Regions of Excess 10-TeV Cosmic Rays, Phys. Rev. Lett., 101, 221101, doi:10.1103/PhysRevLett.101.221101, 2008.

Abdo, A. A., Allen, B. T., Aune, T., Berley, D., Casanova, S., Chen, C., Dingus, B. L., Ellsworth, R. W., Fleysher, L., Fleysher, R., Gonzalez, M. M., Goodman, J. A., Hoffman, C. M., Hopper, B., Hüntemeyer, P. H., Kolterman, B. E., Lansdell, C. P., Linnemann, J. T., McEnery, J. E., Mincer, A. I., Nemethy, P., Noyes, D., Pretz, J., Ryan, J. M., Saz Parkinson, P. M., Shoup, A., Sinnis, G., Smith, A. J., Sullivan, G. W., Vasileiou, V., Walker, G. P., Williams, D. A., and Yodh, G. B.: The Large-Scale Cosmic-Ray Anisotropy as Observed with Milagro, Astrophys. J., 698, 2121, doi:10.1088/0004-637X/698/2/2121, 2009.

Aglietta, M., Alekseenko, V. V., Alessandro, B., Antonioli, P., Arneodo, F., Bergamasco, L., Bertaina, M., Bonino, R., Castellina, A., Chiavassa, A., D’Ettorre Piazzoli, B., Di Sciascio, G., Fulgione, W., Galeotti, P., Ghia, P. L., Iacovacci, M., Mannocchi, G., Morello, C., Navarra, G., Saavedra, O., Stamerra, A., Trinchero, G. C., Valchierotti, S., Vallania, P., Vernetto, S., and Vigorito, C.: Evolution of the Cosmic-Ray Anisotropy Above $1014 \mathrm{eV}$, Astrophys. J. Lett., 692, L130, doi:10.1088/0004-637X/692/2/L130, 2009.

Ahlers, M.: Anomalous Anisotropies of Cosmic Rays from Turbulent Magnetic Fields, Phys. Rev. Lett., 112, 021101, doi:10.1103/PhysRevLett.112.021101, 2014.

Amenomori, M., Ayabe, S., Cui, S. W., et al. (The Tibet AS $\gamma$ Collaboration): Large-Scale Sidereal Anisotropy of Galactic Cosmic-Ray Intensity Observed by the Tibet Air Shower Array, Astrophys. J. Lett., 626, L29-L32, 2005.

Amenomori, M., Ayabe, S., Bi, X. J., et al. (The Tibet AS $\gamma$ Collaboration): Anisotropy and Corotation of Galactic Cosmic Rays, Science, 314, 439-443, 2006.

Amenomori, M., Ayabe, S., Bi, X. J., Chen, D., Cui, S. W., Danzengluobu, D., Ding, L. K., Ding, X. H., Feng, C. F., Feng, Z., Feng, Z. Y., Gao, X. Y., Geng, Q. X., Guo, H. W., He, H. H., He, M., Hibino, K., Hotta, N., Hu, H., Hu, H. B., Huang, J., Huang, Q., Jia, H. Y., Kajino, F., Kasahara, K., Katayose, Y., Kato, C., Kawata, K., Labaciren, L., Le, G. M., Li, A. F., Li, J. Y., Lou, Y.-Q., Lu, H., Lu, S. L., Meng, X. R., Mizutani, K., Mu, J., Munakata, K., Nagai, A., Nanjo, H., Nishizawa, M., Ohnishi, M., Ohta, I., Onuma, H., Ouchi, T., Ozawa, S., Ren, J. R., Saito, T., Saito, T. Y., Sakata, M., Sako, T. K., Sasaki, T., Shibata, M., Shiomi, A., Shirai, T., Sugimoto, H., Takita, M., Tan, Y. H., Tateyama, N., Torii, S., Tsuchiya, H., Udo, S., Wang, B., Wang, H., Wang, X., Wang, Y. G., Wu, H. R., Xue, L., Yamamoto, Y., Yan, C. T., Yang, X. C., Yasue, S., Ye, Z. H., Yu, G. C., Yuan, A. F., Yuda, T., Zhang, H. M., Zhang, J. L., Zhang, N. J., Zhang,
X. Y., Zhang, Y., Zhang, Y., Zhaxisangzhu, Y., and Zhou, X. X.: Implication of the sidereal anisotropy of $5 \mathrm{TeV}$ cosmic ray intensity observed with the Tibet III air shower array, in: Turbulence and Nonlinear Processes in Astrophysical Plasma, edited by: Shaikh, D. and Zank, G. P., American Institute of Physics Conference Series, 283-289, doi:10.1063/1.2778976, 2007.

Amenomori, M., Bi, X. J., Chen, D., Cui, S. W., Danzengluobu, D., Ding, L. K., Ding, X. H., Fan, C., Feng, C. F., Feng, Z., Feng, Z. Y., Gao, X. Y., Geng, Q. X., Gou, Q. B., Guo, H. W., He, H. H., He, M., Hibino, K., Hotta, N., Hu, H., Hu, H. B., Huang, J., Huang, Q., Jia, H. Y., Jiang, L., Kajino, F., Kasahara, K., Katayose, Y., Kato, C., Kawata, K., Labaciren, L., Le, G. M., Li, A. F., Li, H. C., Li, J. Y., Liu, C., Lou, Y., Lu, H., Meng, X. R., Mizutani, K., Mu, J., Munakata, K., Nagai, A., Nanjo, H., Nishizawa, M., Ohnishi, M., Ohta, I., Ozawa, S., Saito, T., Saito, T. Y., Sakata, M., Sako, T. K., Shibata, M., Shiomi, A., Shirai, T., Sugimoto, H., Takita, M., Tan, Y. H., Tateyama, N., Torii, S., Tsuchiya, H., Udo, S., Wang, B., Wang, H., Wang, Y., Wang, Y. G., Wu, H. R., Xue, L., Yamamoto, Y., Yan, C. T., Yang, X. C., Yasue, S., Ye, Z. H., Yu, G. C., Yuan, A. F., Yuda, T., Zhang, H. M., Zhang, J. L., Zhang, N. J., Zhang, X. Y., Zhang, Y., Zhang, Y., Zhang, Y., Zhaxisangzhu, Y., Zhou, X. X., and Kota, J.: Largescale sidereal anisotropy of multi-TeV galactic cosmic rays and the heliosphere, arXiv0909.1026, 2009.

Amenomori, M., Bi, X. J., Chen, D., et al. (The Tibet AS $\gamma$ Collaboration): Time Dependence of Loss-Cone Amplitude measured with the Tibet Air-Shower Array, in: Proc. 32nd ICRC, Beijing, China, 2011.

Bartoli, B., Bernardini, P., Bi, X. J., et al. (ARGO-YBJ Collaboration): Medium scale anisotropy in the $\mathrm{TeV}$ cosmic ray flux observed by ARGO-YBJ, Phys. Rev. D, 88, 082001, doi:10.1103/PhysRevD.88.082001, 2013.

Benzvi, S., Fiorino, D., and Sparks, K. (for the hawc collaboration): Observations of the Anisotropy of Cosmic Rays with HAWC, in: Proc. 33rd ICRC, Rio de Janeiro, Brazil, 2013.

BenZvi, S.: Observations of the anisotropy of cosmic rays at TeVPeV, ASTRA Proc., 1, 33-37, doi:10.5194/ap-1-33-2014, 2014.

Biermann, P. L., Becker Tjus, J., Seo, E.-S., and Mandelartz, M.: Cosmic-Ray Transport and Anisotropies, Astrophys. J., 768, 124, doi:10.1088/0004-637X/768/2/124, 2013.

Blasi, P. and Amato, E.: Diffusive propagation of cosmic rays from supernova remnants in the Galaxy. II: anisotropy, JCAP, 1, 11, doi:10.1088/1475-7516/2012/01/011, 2012.

Borovikov, S. N., Pogorelov, N. V., Zank, G. P., and Kryukov, I. A.: Consequences of the Heliopause Instability Caused by Charge Exchange, Astrophys. J., 682, 1404, doi:10.1086/589634, 2008.

Cui, S.: Study on large-scale CR anisotropy with ARGOYBJ experiment, International Cosmic Ray Conference, doi:10.7529/ICRC2011/V01/0041, 2011.

de Jong, J.: Observations of Large Scale Sidereal Anisotropy in 1 and $11 \mathrm{TeV}$ cosmic rays from the MINOS experiment, in: Proc. 32nd ICRC, Beijing, China, 11-18 August 2011.

Desiati, P. and Lazarian, A.: Cosmic rays and stochastic magnetic reconnection in the heliotail, Nonlin. Processes Geophys., 19, 351-364, doi:10.5194/npg-19-351-2012, 2012.

Desiati P. and Lazarian A.: Anisotropy of TeV Cosmic Rays and Outer Heliospheric Boundaries, Astrophys. J., 762, 44, doi:10.1088/0004-637X/762/1/44, 2013. 
Di Sciascio, G.: Measurement of Cosmic Ray Spectrum and Anisotropy with ARGO-YBJ, EPJ Web Conf., 52, 04004, doi:10.1051/epjconf/20125204004, 2013.

Drury, L. O'C.: The problem of small angular scale structure in the cosmic ray anisotropy data, in: Proc. 33rd ICRC, Rio de Janeiro, Brazil, 2013.

Effenberger, F., Fichtner, H., Scherer, K., and Büsching, I.: Anisotropic diffusion of Galactic cosmic ray protons and their steady-state azimuthal distribution, Astron. Astrophys., 547, A120, doi:10.1051/0004-6361/201220203, 2012.

Erlykin, A. D. and Wolfendale, A. W.: The Anisotropy of Galactic Cosmic Rays as a Product of Stochastic Supernova Explosions, Astropart. Phys., 25, 183-194, 2006.

Frisch, P. C., Redfield, S., and Slavin, J. D.: The Interstellar Medium Surrounding the Sun, Annu. Rev. Astron. Astr., 49, 237-279, doi:10.1146/annurev-astro-081710-102613, 2011.

Funsten, H. O., Allegrini, F., Crew, G. B., DeMajistre, R., Frisch, P. C., Fuselier, S. A., Gruntman, M., Janzen, P., McComas, D. J., Möbius, E., Randol, B., Reisenfeld, D. B., Roelof, E. C., and Schwadron, N. A.: Structures and Spectral Variations of the Outer Heliosphere in IBEX Energetic Neutral Atom Maps, Science, 326, 964-966, doi:10.1126/science.1180927, 2009.

Gaisser, T. K., Stanev, T., and Tilav, S.: Cosmic ray energy spectrum from measurements of air showers, Frontiers of Physics, 8, 748758, doi:10.1007/s11467-013-0319-7, 2013.

Giacinti, G. and Sigl, G.: Local Magnetic Turbulence and TeVPeV Cosmic Ray Anisotropies, Phys. Rev. Lett., 109, 071101, doi:10.1103/PhysRevLett.109.071101, 2012.

Guillian, G., Hosaka, J., Ishihara, K., et al. (Super-Kamiokande Collaboration): Observation of the anisotropy of $10 \mathrm{TeV}$ primary cosmic ray nuclei flux with the Super-Kamiokande-I detector, Phys. Rev. D, 75, 062003, doi:10.1103/PhysRevD.75.062003, 2007.

Hall, D. L., Munakata, K., Yasue, S., Mori, S., Kato, C., Koyama, M., Akahane, S., Fujii, Z., Fujimoto, K., Humble, J. E., Fenton, A. G., Fenton, K. B., and Duldig, M. L.: Gaussian analysis of two hemisphere observations of galactic cosmic ray sidereal anisotropies, J. Geophys. Res., 104, 6737-6749, 1999.

Haverkorn, H., Gaensler, B. M., Brown, J. C., Bizunok, N. S., McClure-Griffiths, N. M., Dickey, J. M., and Green, A. J.: Enhanced small-scale Faraday rotation in the Galactic spiral arms, Astrophys. J. Lett., 637, L33, doi:10.1086/500543, 2006.

Heerikhuisen, J., et al.: Heerikhuisen, J., Pogorelov, N. V., Zank, G. P., Crew, G. B., Frisch, P. C., Funsten, H. O., Janzen, P. H., McComas, D. J., Reisenfeld, D. B., and Schwadron, N. A.: PickUp Ions in the Outer Heliosheath: A Possible Mechanism for the Interstellar Boundary EXplorer Ribbon, Astrophys. J. Lett., 708, L126, doi:10.1088/2041-8205/708/2/L126, 2010.

Kumar, R. and Eichler, D.: Large-scale Anisotropy of TeV-band Cosmic Rays Rahul Kumar and David Eichler, Astrophys. J., 785, 129, doi:10.1088/0004-637X/785/2/129, 2014.

Lazarian, A. and Desiati, P.: Magnetic Reconnection as the Cause of Cosmic Ray Excess from the Heliospheric Tail, Astrophys. J., 722, 188, doi:10.1088/0004-637X/722/1/188, 2010.

Lazarian, A. and Yan, H.: Superdiffusion of Cosmic Rays: Implications for Cosmic Ray Acceleration, Astrophys. J., 784, 38, doi:10.1088/0004-637X/784/1/38, 2014.
Mizoguchi, Y., Munakata, K., Takita, M., and Kota, J.: The sidereal anisotropy of multi-TeV cosmic rays in an expanding Local Interstellar Cloud, in: 31st International Cosmic Ray Conference, Lodz, Poland, 2009.

Munakata, K., Mizoguchi, Y., Kato, C., Yasue, S., Mori, S., Takita, M., and Kóta, J.: Solar Cycle Dependence of the Diurnal Anisotropy of $0.6 \mathrm{TeV}$ Cosmic-ray Intensity Observed with the Matsushiro Underground Muon Detector, Astrophys. J., 712, 1100, doi:10.1088/0004-637X/712/2/1100, 2010.

Nagashima, K., Fujimoto, K., and Jacklyn, R. M.: Galactic and heliotail-in anisotropies of cosmic rays as the origin of sidereal daily variation in the energy region $<104 \mathrm{GeV}$, J. Geophys. Res., 103, 17429-17440, doi:10.1029/98JA01105, 1998.

Pohl, M. and Eichler, D.: Understanding TeV-band CosmicRay Anisotropy, Astrophys. J., 766, 4 doi:10.1088/0004637X/766/1/4, 2013.

Pogorelov, N. V., Borovikov, S. N., Zank, G. P., and Ogino, T.: Three-Dimensional Features of the Outer Heliosphere Due to Coupling Between the Interstellar and Interplanetary Magnetic Fields. III. The Effects of Solar Rotation and Activity Cycle, Astrophys. J., 696, 1478, doi:10.1088/0004-637X/696/2/1478, 2009.

Pogorelov, N. V., Suess, S. T., Borovikov, S. N., Ebert, R. W., McComas, D. J., and Zank, G. P.: Three-dimensional Features of the Outer Heliosphere due to Coupling between the Interstellar and Interplanetary Magnetic Fields. IV. Solar Cycle Model Based on Ulysses Observations, Astrophys. J., 772, 2, doi:10.1088/0004637X/772/1/2, 2013.

Ptuskin, V.: Propagation of galactic cosmic rays, Astropart. Phys., 39, 44-51, doi:10.1016/j.astropartphys.2011.11.004, 2012.

Santander, M., Desiati, P., BenZvi, S., and Westerhoff, S.: Observations of the Anisotropy of Cosmic Rays with HAWC, in: Proc. 33rd ICRC, Rio de Janeiro, Brazil, 2013a.

Santander, M., Desiati, P., Gurtner, M., Kampert, K.-H., et al.: Observations of the Anisotropy of Cosmic Rays with HAWC, in: Proc. 33rd ICRC, Rio de Janeiro, Brazil, 2013b.

McComas, D. J., Alexashov, D., Bzowski, M., Fahr, H., Heerikhuisen, J., Izmodenov, V., Lee, M. A., Möbius, E., Pogorelov, N., Schwadron, N. A., and Zank, G. P.: The Heliosphere's Interstellar Interaction: No Bow Shock, Science, 336, 1291, doi:10.1126/science.1221054, 2012.

Schwadron, N. A., Bzowski, M., Crew, G. B., Gruntman, M., Fahr, H., Fichtner, H., Frisch, P. C., Funsten, H. O., Fuselier, S., Heerikhuisen, J., Izmodenov, V., Kucharek, H., Lee, M., Livadiotis, G., McComas, D. J., Moebius, E., Moore, T., Mukherjee, J., Pogorelov, N. V., Prested, C., Reisenfeld, D., Roelof, E., and Zank, G. P.: Comparison of Interstellar Boundary Explorer Observations with 3D Global Heliospheric Models, Science, 326, 966-968, doi:10.1126/science.1180986, 2009.

Schwadron, N. A., Adams, F. C., Christian, E. R., Desiati, P., Frisch, P., Funsten, H. O., Jokipii, J. R., McComas, D. J., Moebius, E., and Zank, G. P.: Global Anisotropies in TeV Cosmic Rays Related to the Sun's Local Galactic Environment from IBEX, Science, 343, 988-990, doi:10.1126/science.1245026, 2014.

Zhang, M., Zuo, P., and Pogorelov, N. V.: Heliospheric Influence on the Anisotropy of TeV Cosmic Rays, Astrophys. J., 790, 5, doi:10.1088/0004-637X/790/1/5, 2014. 
Sveshnikova, L. G., Strelnikova, O. N., and Ptuskin, V. S.: Spectrum and anisotropy of cosmic rays at $\mathrm{TeV}-\mathrm{PeV}$-energies and contribution of nearby sources, Astropart. Phys., 50, 33-46, 2013.
Yan H. and Lazarian A.: Cosmic-Ray Propagation: Nonlinear Diffusion Parallel and Perpendicular to Mean Magnetic Field, Astrophys. J., 673, 942, doi:10.1086/524771, 2008. 